\title{
Non-leptonic kaon decays at large $N_{c}$
}

\author{
Andrea Donini ${ }^{1, \star}$, Pilar Hernández ${ }^{1}$, Carlos Pena ${ }^{2}$, and Fernando Romero-López ${ }^{1,3}$ \\ ${ }^{1}$ Instituto de Física Corpuscular (CSIC-UVEG), E-46071 Valencia, Spain \\ ${ }^{2}$ Departamento de Física Teórica and Instituto de Física Teórica UAM-CSIC, Universidad Autónoma de \\ Madrid, E-28049 Madrid, Spain \\ ${ }^{3} \mathrm{Helmholtz} \mathrm{Institut} \mathrm{für} \mathrm{Strahlen-} \mathrm{und} \mathrm{Kernphysik,} \mathrm{University} \mathrm{of} \mathrm{Bonn,} \mathrm{Bonn,} \mathrm{Germany}$
}

\begin{abstract}
We study the scaling with the number of colors $N_{c}$ of the weak amplitudes mediating kaon mixing and decay, in the limit of light charm masses $\left(m_{u}=m_{d}=m_{s}=\right.$ $m_{c}$ ). The amplitudes are extracted directly on the lattice for $N_{c}=3-7$ (with preliminar results for $N_{c}=8$ and 17) using twisted mass QCD. It is shown that the (sub-leading) $1 / N_{c}$ corrections to $\hat{B}_{K}$ are small and that the naive $N_{c} \rightarrow \infty$ limit, $\hat{B}_{K}=3 / 4$, seems to be recovered. On the other hand, the $O\left(1 / N_{c}\right)$ corrections in $K \rightarrow \pi \pi$ amplitudes (derived from $K \rightarrow \pi$ matrix elements) are large and fully anti-correlated in the $I=0$ and $I=2$ channels. This may have some implications for the understanding of the $\Delta I=1 / 2$ rule.
\end{abstract}

\section{Introduction}

The prediction of flavour violating processes involving kaons remains elusive. In particular, there is still no satisfactory explanation of the striking $\Delta I=1 / 2$ "rule" for which $\Delta I=1 / 2$ decays of a kaon into two pions dominate over $\Delta I=3 / 2$ decays (see, however, Refs. [1, 2]), nor a reliable prediction of the parameter that controls direct vs. indirect CP-violation in kaon mixing, $\epsilon^{\prime} / \epsilon$ [3]. Few attempts have been made at these difficult observables, and the systematic uncertainties in the existing results remain large. At the same time, however, a rather precise determination of the related $K-\bar{K}$ mixing amplitude (given by $\hat{B}_{K}$ ) has emerged [4-9].

In [1], the results of the most ambitious lattice computation of $K \rightarrow \pi \pi$ to date were presented, and a significant $\Delta I=1 / 2$ dominance was observed. It was noted that the $\Delta I=1 / 2$ rule seems to be originating in an approximate cancellation of the two diagrams (color connected, $C$, and color disconnected, $D$ ) contributing to the $\Delta I=3 / 2$ amplitude. Roughly speaking, the relative weight of $\Delta I=1 / 2$ $K \rightarrow \pi \pi$ decays with respect to $\Delta I=3 / 2$ ones is governed by the ratio $(|C|+|D|) /(|C|-|D|)$. As in Ref. [1] and in its update [2] it was found $|C| \sim 0.8|D|$, a significant enhancement of this ratio was observed at the non-perturbative level. This result, combined with the known perturbative enhancement of the ratio due to Wilson coefficients connecting short- and long-distance QCD matrix elements $[10,11]$, would then explain the $\Delta I=1 / 2$ rule. Unfortunately, it is not possible to isolate these two contributions physically, so it is not clear what to extract from this finding. In the large $N_{c}$ expansion [12], however, this is possible since the leading scaling in $N_{c}$ of the contributions is different. The cancellation can therefore be phrased in terms of the sign and size of the $1 / N_{c}$ corrections in the

^ Speaker, e-mail: donini@ific.uv.es 
isospin amplitudes. In fact, it was in the context of phenomenological approaches using the large $N_{c}$ expansion where the opposite sign of these contributions was first pointed out [13]. Notice that, at the leading order in the $1 / N_{c}$ expansion, the connected diagram $C$ is expected to be $|C| \sim 1 / N_{c} \times|D|$. The numerical results of Refs. [1,2] are, therefore, pointing to a possible large deviation of the ratio above from the naive $1 / N_{c}$ expectations. As there is a strong correlation between the $\Delta I=3 / 2$ amplitude and $\hat{B}_{K}$, this suggest that the same cancellation in the former should be affecting the latter, suggesting a value of $\hat{B}_{K}$ significantly smaller than the $N_{c} \rightarrow \infty$ value. A study of the issue of deviations from the naïve factorization approximation to $K \rightarrow \pi \pi$ amplitudes can be found in [14].

The large $N_{c}$ limit of QCD has been invoked in many phenomenological approaches to this problem (some relevant references are $[13,15-18]$ ). This seems counter-intuitive since the strict large $N_{c}$ limit of the $\Delta I=1 / 2$ rule fails completely. The predictions therefore rely on significant sub-leading $N_{c}$ effects, a computation that poses formidable difficulties. As a result, these approaches typically involve further approximations beyond the strict large- $N_{c}$ expansion. The goal of Ref. [19], summarized in this proceeding, was to study from first principles the $N_{c}$ dependence of certain $\Delta S=1$ and $\Delta S=2$ amplitudes to check their scaling with the number of colors.

\section{Strategy and simulation details}

In order to study the non-perturbative $N_{c}$ dependence of $K \rightarrow \pi \pi$ amplitudes, we have followed the strategy outlined in Ref. [20]: we have measured $K-\pi$ and $K-\bar{K}$ matrix elements mediated by the fourfermion current-current operators on the lattice, varying the number of colors $N_{c}$ between $N_{c}=3$ and $N_{c}=7$ (some preliminary results for $N_{c}=8$ and $N_{c}=17$ will be also shown). In the SU(4)-flavour limit ${ }^{1}, m_{c}=m_{u}=m_{d}=m_{s}$, these amplitudes fix $\hat{B}_{K}$ (up to $\mathrm{SU}(3)$ flavour breaking effects by quark masses) and, up to chiral corrections, also the $\Delta I=3 / 2[23,24]$ and $\Delta I=1 / 2[20,25]$ contributions to the non-leptonic kaon decays $K \rightarrow \pi \pi$. The weak Hamiltonian that mediates CP-conserving $\Delta S=1$ transitions, in terms of four-fermion operators at the electroweak scale, $\mu \simeq M_{W}$, takes the following simple form in this limit:

$$
H_{\mathrm{w}}^{\Delta S=1}=\int d^{4} x \frac{g_{\mathrm{w}}^{2}}{4 M_{W}^{2}} V_{u s}^{*} V_{u d} \sum_{\sigma= \pm} k^{\sigma}(\mu) \bar{Q}^{\sigma}(x, \mu),
$$

where $g_{\mathrm{w}}^{2}=4 \sqrt{2} G_{\mathrm{F}} M_{W}^{2}$. Only two four-quark operators of dimension six can appear with the correct symmetry properties under the flavour symmetry group $\mathrm{SU}(4)_{\mathrm{L}} \times \mathrm{SU}(4)_{\mathrm{R}}$, namely

$$
\bar{Q}^{ \pm}(x, \mu)=Z_{Q}^{ \pm}(\mu)\left(J_{\mu}^{s u}(x) J_{\mu}^{u d}(x) \pm J_{\mu}^{s d}(x) J_{\mu}^{u u}(x)-[u \leftrightarrow c]\right),
$$

where $J_{\mu}$ is the left-handed current, $J_{\mu}^{\alpha \beta}=\left(\bar{\psi}_{\alpha} \gamma_{\mu} P_{-} \psi_{\beta}\right), P_{ \pm}=\frac{1}{2}\left(\mathbf{1} \pm \gamma_{5}\right)$, and parentheses around quark bilinears indicate that they are traced over spin and colour. Eventually, $Z_{Q}^{ \pm}(\mu)$ is the renormalisation constant of the bare operator $Q^{ \pm}(x)$ computed in some regularisation scheme as, for example, the lattice. There are other bilinear operators of lower dimensionality that could mix with those above: however, their contribution vanishes in the SU(4) limit [20]. Notice that this is, indeed, the limit where the cancellation of Ref. [1] can be more clearly isolated.

The operators $\bar{Q}^{\sigma}(\mu)$ are renormalised at a scale $\mu$ in some renormalisation scheme, being their $\mu$-dependence exactly cancelled by that of the Wilson coefficients $k^{\sigma}(\mu)$. The renormalisation group

\footnotetext{
${ }^{1}$ We are aware to miss, in this way, the effect of the decoupling of a heavy charm, which was originally argued to be the origin of the $\Delta I=1 / 2$ rule [21] (something not confirmed by recent non-perturbative studies [3, 22]).
} 
invariant (RGI) operators are defined eploiting this fact to eliminate their $\mu$ - and scheme-dependence:

$$
\hat{Q}^{\sigma} \equiv \hat{c}^{\sigma}(\mu) \bar{Q}^{\sigma}(\mu), \quad \hat{c}^{\sigma}(\mu) \equiv\left(\frac{N_{c}}{3} \frac{g^{2}(\mu)}{4 \pi}\right)^{-\frac{\gamma_{0}^{\sigma}}{2 b_{0}}} \times \exp \left\{-\int_{0}^{g(\mu)} \mathrm{d} g\left[\frac{\gamma^{\sigma}(g)}{\beta(g)}-\frac{\gamma_{0}^{\sigma}}{b_{0} g}\right]\right\},
$$

where $g(\mu)$ is the running coupling and $\beta(g)=-g^{3} \sum_{n} b_{n} g^{2 n}, \gamma^{\sigma}(g)=-g^{2} \sum_{n} \gamma_{n}^{\sigma} g^{2 n}$ are the $\beta$-function and the anomalous dimension, respectively. The one- and two-loop coefficients of the $\beta$-function, $b_{0}$ and $b_{1}$, and the one-loop coefficient of the anomalous dimensions, $\gamma_{0}^{ \pm}$, are renormalisation schemeindependent and can be found in Refs. [26-30] and [10, 11], respectively. The normalisation of $\hat{c}^{\sigma}(\mu)$ coincides with the most popular one for $N_{c}=3$, whilst using the 't Hooft coupling $\lambda=N_{c} g^{2}(\mu)$ in the first factor instead of the usual coupling, so that the large $N_{c}$ limit is well defined.

We can rewrite the Hamiltonian in terms of RGI quantities, which no longer depend on the scale:

$$
\hat{k}^{\sigma} \equiv \frac{k^{\sigma}(\mu)}{\hat{c}^{\sigma}(\mu)}, \quad \hat{k}^{\sigma} \hat{Q}^{\sigma}=\left[\frac{k^{\sigma}\left(M_{W}\right)}{\hat{c}^{\sigma}\left(M_{W}\right)}\right]\left[\hat{c}^{\sigma}(\mu) \bar{Q}^{\sigma}(\mu)\right]=k^{\sigma}\left(M_{W}\right) U^{\sigma}\left(\mu, M_{W}\right) \bar{Q}^{\sigma}(\mu),
$$

where $\mu$ is a convenient renormalisation scale for the non-perturbative computation of matrix elements of $Q^{ \pm}$, which will be later set to the inverse lattice scale $a^{-1}$. The factor $U^{\sigma}\left(\mu, M_{W}\right)=\hat{c}^{\sigma}(\mu) / \hat{c}^{\sigma}\left(M_{W}\right)$ measures the running of the renormalised operator between the scales $\mu$ and $M_{W}$. In Table 1 we show the RG running factors needed to compute the renormalised $K \rightarrow \pi$ and $K \rightarrow \bar{K}$ matrix elements as a function of the number of colors. In the evaluation of the $\hat{c}^{\sigma}(\mu)$ factors we have used the large $N_{c}$ scaling of $\Lambda_{\mathrm{QCD}}$ found in Ref. [31],

$$
\frac{\Lambda_{\overline{M S}}}{\sqrt{\sigma}}=0.503(2)(40)+\frac{0.33(3)(3)}{N_{c}^{2}} .
$$

The values of the normalisation coefficients $\hat{c}^{ \pm}\left(a^{-1}\right)$ and of the running of the renormalised operators from the scale of lattice computations, $\mu=a^{-1}$, to the scale of the effective theory, $M_{W}$, have been computed using perturbative results at two loops in the RI scheme [32, 33]. This implies relying on perturbation theory at scales above $\mu=a^{-1} \sim 2 \mathrm{GeV}$.

Table 1. Perturbative RG running factors. $U^{\sigma}$ and $k^{\sigma}$ are computed using the two-loop $\overline{\mathrm{MS}}$ coupling (with $\Lambda_{\overline{M S}}$ taken from eq. (5) from ref. [31]).

\begin{tabular}{ccccccccc}
\hline \hline$N_{c}$ & $\hat{k}^{+}$ & $k^{+}\left(M_{W}\right)$ & $U^{+}\left(a^{-1}, M_{W}\right)$ & $\hat{c}^{+}\left(a^{-1}\right)$ & $\hat{k}^{-}$ & $k^{-}\left(M_{W}\right)$ & $U^{-}\left(a^{-1}, M_{W}\right)$ & $\hat{c}^{-}\left(a^{-1}\right)$ \\
\hline 3 & 0.642 & 1.030 & 0.875 & 1.404 & 2.398 & 0.940 & 1.319 & 0.517 \\
4 & 0.658 & 1.025 & 0.895 & 1.394 & 1.998 & 0.958 & 1.210 & 0.580 \\
5 & 0.679 & 1.021 & 0.910 & 1.368 & 1.780 & 0.968 & 1.156 & 0.620 \\
6 & 0.700 & 1.018 & 0.921 & 1.340 & 1.643 & 0.974 & 1.124 & 0.666 \\
7 & 0.719 & 1.016 & 0.930 & 1.315 & 1.550 & 0.978 & 1.103 & 0.696 \\
\hline 8 & 0.736 & 1.015 & 0.938 & 1.293 & 1.480 & 0.981 & 1.088 & 0.721 \\
17 & 0.827 & 1.007 & 0.968 & 1.178 & 1.238 & 0.992 & 1.038 & 0.832 \\
\hline \hline
\end{tabular}

As specified above, our goal is to compute the $K \rightarrow \pi$ amplitudes mediated by $H_{\mathrm{w}}^{\Delta S=1}$. The hadronic contribution is encoded in the ratios of the following matrix elements:

$$
\hat{R}^{ \pm} \equiv \frac{\left\langle\pi\left|\hat{Q}^{ \pm}\right| K\right\rangle}{f_{K} f_{\pi} m_{K} m_{\pi}}=\hat{c}^{ \pm}(\mu) Z_{R}^{ \pm}(\mu) R^{ \pm},
$$

where $Z_{R}^{ \pm}(\mu)$ are the renormalisation factors for the ratios and $R^{ \pm}$is the ratio of matrix elements of bare operators. The ratio of the two isospin amplitudes $i A_{0,2} e^{i \delta_{0,2}} \equiv\left\langle(\pi \pi)_{0,2}\left|H_{W}\right| K_{0}\right\rangle$ (where the subindex 
refers to the final isospin state) can be related in chiral perturbation theory in the GIM limit to the $K \rightarrow \pi$ amplitudes $A^{ \pm} \equiv \hat{k}^{ \pm} \hat{R}^{ \pm}[20]$ as follows:

$$
\frac{A_{0}}{A_{2}}=\frac{1}{\sqrt{2}}\left(\frac{1}{2}+\frac{3}{2} \frac{A^{-}}{A^{+}}\right),
$$

from which we can see that the large enhancement of the ratio $\left|A_{0} / A_{2}\right| \sim 22$ is related, in this limit, to a large value of the ratio of the amplitudes $A^{-} / A^{+}$(up to chiral corrections). In the SU(3) limit $m_{s}=m_{d}=m_{u}$, the kaon mixing amplitude given by $\hat{B}_{K}$ is also related to $R^{+}, \hat{B}_{K}=\frac{3}{4} \hat{R}^{+}$(something not true outside of the SU(3) limit, due to large chiral corrections [23, 24]). The relation between $K \rightarrow \pi \pi$ and $K \rightarrow \pi$ amplitudes, computed up to one loop in ChPT in the leading-log approximation, is:

$$
\left.\frac{\left\langle\pi^{+} \pi^{0}\left|H_{W}\right| K\right\rangle}{m_{K}^{2}-m_{\pi}^{2}}\right|_{m_{s}=m_{d}}=\frac{i F}{\sqrt{2}} A^{+} G_{F} V_{u d} V_{u s}^{*},
$$

where $F$ is the decay constant in the chiral limit and $A^{+}$contains one loop corrections. This shows that, in this approximation, the $1 / N_{c}$ corrections in the physical amplitude are fixed ${ }^{2}$ by those in $A^{+}$.

On the lattice, the ratios $\hat{R}^{ \pm}$are extracted as follows:

$$
R^{ \pm} \lim _{\substack{z_{0}-x_{0} \rightarrow \infty \\ y_{0}-z_{0} \rightarrow \infty}} \frac{\sum_{\mathbf{x}, \mathbf{y}}\left\langle P^{d u}(y) Q^{ \pm}(z) P^{u s}(x)\right\rangle}{\sum_{\mathbf{x}, \mathbf{y}}\left\langle P^{d u}(y) A_{0}^{u d}(z)\right\rangle\left\langle A_{0}^{s u}(z) P^{u s}(x)\right\rangle},
$$

where $P^{a b}(x)=\bar{\psi}^{a}(x) \gamma_{5} \psi^{b}(x)$, and $A_{0}^{a b}(x)=Z_{\mathrm{A}} \bar{\psi}^{a}(x) \gamma_{0} \gamma_{5} \psi^{b}(x)$. We have computed the renormalised ratios $\hat{R}^{ \pm}$in the quenched approximation. This does not modify the leading large $N_{c}$ result, but it can modify the first sub-leading $1 / N_{c}$ corrections (we plan to address this issue in further studies). We have implemented the required correlation functions in the source code first developed in [38] and further optimized in [39]. The number of colors and the lattice size in the time direction are given in the first two columns of Table 2. The spatial volume, $L / a=16$, is kept fixed in all simulations (but for $N_{c}=17$, for which $L / a=12$, as in Ref. [40]). Following [40] the bare coupling, $\beta=2 N_{c} / g_{0}^{2}$, is tuned with $N_{c}$ in such a way that the string tension is $a \sqrt{\sigma} \simeq 0.2093$ for all $N_{c}$; this results in $a \simeq 0.093 \mathrm{fm}$ with $\sigma=1 \mathrm{GeV} / \mathrm{fm}$. The bare 't Hooft coupling $\lambda$ for $N_{c} \in[3,7]$ is well described by the scaling:

$$
\lambda=N_{c} g_{0}^{2}=2.775(3)+\frac{1.90(3)}{N_{c}^{2}} .
$$

The coupling $\beta$ as a function of $N_{c}$ is given in the third column of Table 2. The gauge action is the standard plaquette action. On the other hand, in order to preserve the multiplicative renormalisation of $Q^{ \pm}$, while avoiding the high computational cost of a simulation with exactly chiral lattice fermions, we use a Wilson twisted-mass fermion regularisation [41, 42]. This allows to devise a formulation of valence quarks that not only preserves good renormalisation properties, but also prevents the appearance of linear cutoff effects in $a$ [43]. The full-twist condition amounts to having a vanishing current quark mass $m_{\text {PCAC }}$ from the axial Takahashi-Ward identity in so-called twisted quark field variables. The value of $a m_{\mathrm{PCAC}}$ in our simulations is given in the fourth column of Table 2, where we can see that the full-twist condition $a m_{\mathrm{PCAC}}=0$, expected from an accurate tuning of the Wilson critical mass (which we again take from [40]), is satisfied to a varying degree of accuracy; the deviations present are however irrelevant within the precision of our results. The bare quark mass is chosen to provide a pseudoscalar mass not far from the physical kaon mass in all cases (see the fifth column of Table 2).

\footnotetext{
${ }^{2}$ It has been argued, however, that higher-order ChPT effects may have an important impact on $K \rightarrow \pi \pi$ amplitudes at the same order in $1 / N_{c}$ (see, e.g., Refs. [34-37]).
} 
Table 2. Lattice simulation results. Lattice sizes are $(L / a)^{3} \times(T / a)$, with $L / a=16$ throughout ( ${ }^{\star}$ with the only exception of $N_{c}=17$, for which $L / a=12$ ). The twisted bare mass is fixed to $a \mu=0.02$. The lattice spacing is fixed by the string tension: $a \sqrt{\sigma} \simeq 0.2093$ [40]. $m_{\mathrm{PCAC}}$ is the current mass obtained from the axial Takahashi-Ward identity in twisted quark field variables. $m_{\mathrm{PS}}$ is the meson mass in the SU(3) limit. $R^{ \pm}$are our results for the bare ratios given in eq. (9). $Z^{\sigma}\left(a^{-1}\right)$ at one-loop have been extracted from [44, 45].

\begin{tabular}{ccccccccc}
\hline \hline$N_{c}$ & $T / a$ & $\beta$ & $a m_{\mathrm{PCAC}}$ & $a m_{\mathrm{PS}}$ & $R_{\text {bare }}^{+}$ & $R_{\text {bare }}^{-}$ & $Z^{+}\left(a^{-1}\right)$ & $Z^{-}\left(a^{-1}\right)$ \\
\hline 3 & 48 & 6.0175 & $-0.002(14)$ & $0.2718(61)$ & $0.774(21)$ & $1.218(31)$ & 0.983 & 1.059 \\
4 & 48 & 11.028 & $-0.0015(11)$ & $0.2637(39)$ & $0.783(15)$ & $1.198(19)$ & 0.988 & 1.043 \\
5 & 48 & 17.535 & $0.0028(9)$ & $0.2655(31)$ & $0.839(8)$ & $1.145(12)$ & 0.991 & 1.035 \\
6 & 32 & 25.452 & $0.0013(7)$ & $0.2676(28)$ & $0.871(6)$ & $1.125(7)$ & 0.994 & 1.030 \\
7 & 32 & 34.8343 & $-0.0034(6)$ & $0.2819(19)$ & $0.880(5)$ & $1.122(5)$ & 0.996 & 1.026 \\
\hline 8 & 32 & 45.7003 & $-0.0002(6)$ & $0.3065(23)$ & $0.872(4)$ & $1.127(4)$ & 0.997 & 1.024 \\
17 & $24^{\star}$ & 208.45 & $-0.00178(45)$ & $0.2594(9)$ & $0.967(3)$ & $1.039(6)$ & 1.003 & 1.015 \\
\hline \hline
\end{tabular}

Eventually, our results for the bare ratios $R^{ \pm}$defined in eq. (9), computed in the SU(3) limit, are shown in the sixth and seventh columns of the table. The corresponding renormalisation constants, $Z^{ \pm}$, have been computed at one-loop in the RI scheme using the scripts provided in Refs. [44, 45]. Notice that, due to the breaking of chiral symmetry in the adopted regularisation, the axial current requires a finite, $N_{c}$-dependent, renormalisation constant $Z_{A}$ (taken from the same references), that has to be included in the factors $Z^{ \pm}$. The values of $Z^{ \pm}\left(a^{-1}\right)$ are given in the two rightmost columns of Table 2. The results shown for $R^{ \pm}$correspond to $O(100)$ measurements at each value of $N_{c}$, with each measurement taken every 1000 gauge updates. The only exception is the run for $N_{c}=17$, for which we have only 17 measurements taken every 100 gauge updates. We have checked, however, that all measurements are fully decorrelated using the techniques of Ref. [46].

\section{Results}
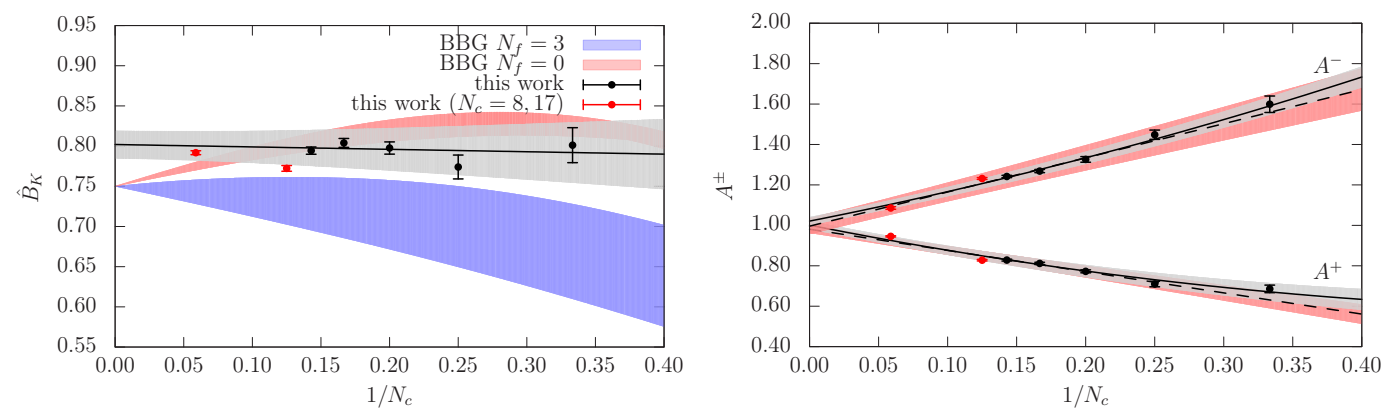

Figure 1. Left: $\hat{B}_{K}$ versus $1 / N_{c}$. The grey band (solid line) is a linear fit to our five data points. The red and blue bands use the model prediction of [15]. Right: $A^{ \pm}$versus $1 / N_{c}$. The grey bands (solid lines) are obtained from the results of the fits to $1 / 2\left(A^{-} \pm A^{+}\right)$in eqs. (11); the red bands (dashed lines) are linear fits including $N_{c}=4-7$ from Table 3. Data points for $N_{c}=8$ and 17 (in red) have been added to the plots, but not used in the fits.

Our results for $\hat{B}_{K}$ as a function of $1 / N_{c}$ are shown in Fig. 1(left) together with a linear fit to the data for $\left.N_{c}=3-7\right]$, represented by a solid black line (results for $N_{c}=8$ and 17 have not been used 
in the fit). The grey band shows the $1 \sigma$ error on the fit. The parameters of the fit are shown in the first two lines of Table 3 for two choices of the data points included, together with the corresponding $p$-values. The third line of the same table shows our result for a quadratic fit to the data. We compare our results with our own evaluation of the predictions of the phenomenological analysis in Ref. [15], represented by a light red band for $N_{f}=3$ and by a blue band for $N_{f}=0$. For $N_{f}=3$ we use the same values for hadronic masses and decay constants as in [15], and derive the decay constant for $N_{c} \neq 3$ by rescaling $F_{K}=F_{K}\left(N_{c}=3\right) \sqrt{N_{c} / 3}$. For $N_{f}=0$ we use as input for the hadronic quantities, including their $N_{c}$ dependence, the interpolating formulae provided in [40], matched to our measured values of $M_{K}$. The band represent the difference between setting the matching scale $M$ in eq. (62) of [15] at $0.6 \mathrm{GeV}$ and at $1 \mathrm{GeV}$; for $N_{f}=0$ it also comprises the uncertainty due to our value of $M_{K}$ not being constant within errors as a function of $N_{c}$. Notice that both theoretical predictions give $\hat{B}_{K}=3 / 4$ in the $N_{c} \rightarrow \infty$ limit. On the other hand, our data (and the preliminar values for $N_{c}=8$ and 17) give a value for $\hat{B}_{K}$ at $N_{c} \rightarrow \infty$ slightly larger than the theoretical expectation. Errors are, however, still too large to draw any conclusion. For example, a significant $O\left(a^{2}\right)$ uncertainty for $R^{+}$can be expected, cf. the $O(10 \%)$ effect for $N_{c}=3, N_{f}=2$ shown by the data of [47] at a lattice spacing comparable to ours.

Table 3. Fit parameters of $A^{ \pm}$assuming a linear (l) or quadratic (q) dependence, and various fit ranges (using only data up to $N_{c}=7$, though). The order at which each coefficient enters in the polynomial ansatz in powers of $1 / N_{c}$ is indicated, alongside with the $p$-value for each fit.

\begin{tabular}{cccccc}
\hline \hline obs & fit & 1 & $1 / N_{c}$ & $1 / N_{c}^{2}$ & $p$-value \\
\hline$\hat{B}_{K}$ & $1, N_{c} \geq 3$ & $0.802(17)$ & $-0.03(10)$ & - & 0.24 \\
& $1, N_{c} \geq 4$ & $0.808(27)$ & $-0.07(16)$ & - & 0.14 \\
& $\mathrm{q}, N_{c} \geq 3$ & $0.788(79)$ & $0.12(78)$ & $-0.3(1.8)$ & 0.12 \\
\hline$A^{+}$ & $1, N_{c} \geq 3$ & $0.956(20)$ & $-0.89(11)$ & - & 0.10 \\
& $1, N_{c} \geq 4$ & $0.981(18)$ & $-1.05(11)$ & - & 0.39 \\
\hline$A^{-}$ & $1, N_{c} \geq 3$ & $0.984(28)$ & $1.77(17)$ & - & 0.21 \\
& $1, N_{c} \geq 4$ & $0.996(39)$ & $1.69(24)$ & - & 0.14 \\
\hline \hline
\end{tabular}

From Fig. 1(left) we can see that the sub-leading $1 / N_{c}$ corrections in $\hat{B}_{K}$ are small (which goes in the direction of the predictions in [15], but not those in [16], that correspond to the chiral limit). The smallness of $1 / N_{c}$ corrections in $\hat{B}_{K}$ is related to the RGI normalization of this quantity, $\hat{c}^{+}\left(a^{-1}\right)$ : the $N_{c}$-dependence of $R^{+}$(see Table 2) is cancelled by the RGI Wilson coefficient $\hat{k}^{+}$(see Table 1). In contrast, the total $K \rightarrow \pi$ amplitudes show very significant sub-leading $1 / N_{c}$ corrections, as shown in Fig. 1(right). In the Figure we present our data for $A^{ \pm}$and the results of a linear (dashed lines) and quadratic (solid lines) fit to the data, obtained using again only data for $N_{c}=3-7$ (preliminar data for $N_{c}=8$ and 17, although not used in the fits, are perfectly compatible with the results obtained for lower values of $N_{c}$ ). The parameters of the linear fit for $A^{+}$and $A^{-}$are shown in the fourth and fifth (sixth and seventh) lines of Table 3 , respectively. We can see that $A^{+}$and $A^{\text {' }}$ are strongly anticorrelated in $N_{c}$ and that their extrapolation at $N_{c} \rightarrow \infty$ is in very good agreement with theoretical expectations (for which $\left|A_{0} / A_{2}\right|_{N_{c} \rightarrow \infty} \sim \sqrt{2}$ ). Notice that, in the GIM limit, the chiral logs have been shown to be fully anti-correlated in $A^{ \pm}$[48] and therefore an extrapolation to the chiral limit using chiral perturbation theory should not change the anti-correlation found here. Unfortunately, the computation of chiral logs in $K \rightarrow(\pi \pi)_{I=0}$ in the GIM limit is not yet available.

Eventually, our results for the combinations $\frac{1}{2}\left(A^{-} \pm A^{+}\right)$, corresponding to the (renormalized) connected $\hat{C}$ and disconnected $\hat{D}$ diagrams, respectively, are shown in Fig. 2. A quadratic fit using 
$N_{c}=3-7$ data gives the following results:

$$
\begin{aligned}
& \hat{C}=\frac{A^{-}-A^{+}}{2}=0.01(2)+\frac{1.35(11)}{N_{c}} \quad(p \text {-value }=0.12), \\
& \hat{D}=\frac{A^{-}+A^{+}}{2}=1.01(3)+\frac{1.08(11)}{N_{c}^{2}} \quad(p \text {-value }=0.81) .
\end{aligned}
$$

Our results show that the sub-leading $1 / N_{c}$ effects cancel in the "disconnected" contribution to $K \rightarrow \pi$, whereas they are the only visible corrections in the "connected" one. In particular, it is clear that a relation as the one expected naively in the large $N_{c}$ expansion, $|\hat{C}| \sim k / N_{c} \times|\hat{D}|$, holds, with a coefficient $k \sim 1.3$ much smaller than what found in Refs. [1,2]. The source of the huge non-perturbative cancellation between "connected" and "disconnected" contributions seem not to arise from a failure of naive $N_{c}$ scaling, but in some other enhancement of the coefficient $k$ that relates the two amplitudes (for example, large $1 / N_{c}$ corrections could be present at the physical point, $m_{s} \gg m_{d}$, as suggested by a large chiral $\log$ ).
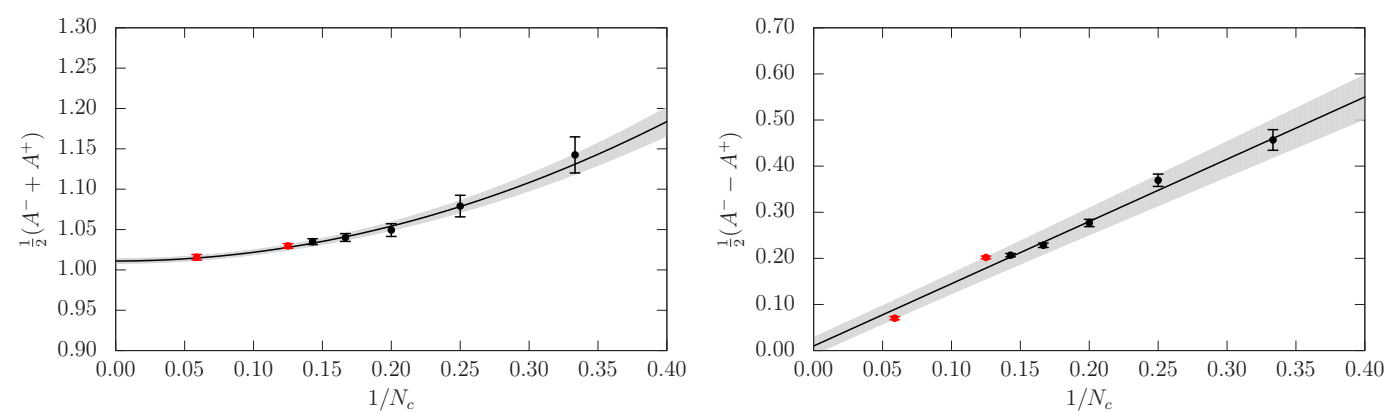

Figure 2. $\frac{A^{-} \pm A^{+}}{2}$ versus $1 / N_{c}$. The bands (solid lines) are quadratic and linear fits in $1 / N_{c}$, respectively. Data points for $N_{c}=8$ and 17 (in red) have been added to the plots, but not used in the fits.

We have not included any systematic error in these results. There are two obvious sources: finite lattice spacing and the quenched approximation. Although it is impossible to quantify those errors, we do not expect them to be larger that those observed at $N_{c}=3$, where they have been studied. The pioneering large- $N_{c}$ study of dynamical QCD in [49] shows that an extension of our work to take into account unquenching effects is feasible.

\section{References}

[1] P.A. Boyle et al. (RBC, UKQCD), Phys. Rev. Lett. 110, 152001 (2013), 1212 . 1474

[2] T. Blum et al., Phys. Rev. D91, 074502 (2015), 1502.00263

[3] Z. Bai et al. (RBC, UKQCD), Phys. Rev. Lett. 115, 212001 (2015), 1505.07863

[4] S. Aoki et al., Eur. Phys. J. C74, 2890 (2014), 1310. 8555

[5] S. Durr et al., Phys. Lett. B705, 477 (2011), 1106.3230

[6] J. Laiho, R.S. Van de Water, PoS LATTICE2011, 293 (2011), 1112.4861

[7] T. Blum et al. (RBC, UKQCD), Phys. Rev. D93, 074505 (2016), 1411.7017

[8] B.J. Choi et al. (SWME), Phys. Rev. D93, 014511 (2016), 1509.00592

[9] N. Carrasco et al. (ETM), Phys. Rev. D92, 034516 (2015), 1505.06639 
[10] M.K. Gaillard, B.W. Lee, Phys. Rev. Lett. 33, 108 (1974)

[11] G. Altarelli, L. Maiani, Phys. Lett. 52B, 351 (1974)

[12] G. 't Hooft, Nucl. Phys. B72, 461 (1974)

[13] A. Pich, E. de Rafael, Phys. Lett. B374, 186 (1996), hep-ph/9511465

[14] N. Carrasco, V. Lubicz, L. Silvestrini (ETM), Phys. Lett. B736, 174 (2014), 1312 . 6691

[15] A.J. Buras, J.M. Gerard, W.A. Bardeen, Eur. Phys. J. C74, 2871 (2014), 1401.1385

[16] S. Peris, E. de Rafael, Phys. Lett. B490, 213 (2000), hep-ph/0006146

[17] T. Hambye, S. Peris, E. de Rafael, JHEP 05, 027 (2003), hep-ph/0305104

[18] V. Cirigliano et al., Rev. Mod. Phys. 84, 399 (2012), 1107.6001

[19] A. Donini, P. Hernandez, C. Pena, F. Romero-Lopez, Phys. Rev. D94, 114511 (2016), 1607.03262

[20] L. Giusti et al., JHEP 11, 016 (2004), hep-lat/0407007

[21] M.A. Shifman, A.I. Vainshtein, V.I. Zakharov, Nucl. Phys. B120, 316 (1977)

[22] E. Endress, C. Pena, Phys. Rev. D90, 094504 (2014), 1402.0827

[23] J.F. Donoghue, E. Golowich, B.R. Holstein, Phys. Lett. 119B, 412 (1982)

[24] J. Bijnens, H. Sonoda, M.B. Wise, Phys. Rev. Lett. 53, 2367 (1984)

[25] L. Giusti et al., Phys. Rev. Lett. 98, 082003 (2007), hep-ph/0607220

[26] D.J. Gross, F. Wilczek, Phys. Rev. Lett. 30, 1343 (1973)

[27] H.D. Politzer, Phys. Rev. Lett. 30, 1346 (1973)

[28] W.E. Caswell, Phys. Rev. Lett. 33, 244 (1974)

[29] D.R.T. Jones, Nucl. Phys. B75, 531 (1974)

[30] E. Egorian, O.V. Tarasov, Teor. Mat. Fiz. 41, 26 (1979), [Theor. Math. Phys.41,863(1979)]

[31] C. Allton, M. Teper, A. Trivini, JHEP 07, 021 (2008), 0803. 1092

[32] M. Ciuchini et al., Nucl. Phys. B523, 501 (1998), hep-ph/9711402

[33] A.J. Buras, M. Misiak, J. Urban, Nucl. Phys. B586, 397 (2000), hep-ph/0005183

[34] T.N. Truong, Phys. Lett. B207, 495 (1988)

[35] N. Isgur, K. Maltman, J.D. Weinstein, T. Barnes, Phys. Rev. Lett. 64, 161 (1990)

[36] J. Kambor, J.H. Missimer, D. Wyler, Phys. Lett. B261, 496 (1991)

[37] E. Pallante, A. Pich, Nucl. Phys. B592, 294 (2001), hep-ph/0007208

[38] L. Del Debbio, A. Patella, C. Pica, Phys. Rev. D81, 094503 (2010), 0805.2058

[39] C. Pica (2016), private communication.

[40] G.S. Bali et al., JHEP 06, 071 (2013), 1304 . 4437

[41] R. Frezzotti, P.A. Grassi, S. Sint, P. Weisz (Alpha), JHEP 08, 058 (2001), hep-lat/0101001

[42] R. Frezzotti, G.C. Rossi, JHEP 08, 007 (2004), hep-lat/0306014

[43] R. Frezzotti, G.C. Rossi, JHEP 10, 070 (2004), hep-lat/0407002

[44] M. Constantinou et al., Phys. Rev. D83, 074503 (2011), 1011.6059

[45] C. Alexandrou et al., Phys. Rev. D86, 014505 (2012), 1201.5025

[46] U. Wolff (ALPHA), Comput. Phys. Commun. 156, 143 (2004), [Erratum: Comput. Phys. Commun.176,383(2007)], hep-lat/0306017

[47] M. Constantinou et al. (ETM), Phys. Rev. D83, 014505 (2011), 1009.5606

[48] P. Hernandez, M. Laine, JHEP 10, 069 (2006), hep-lat/0607027

[49] T. DeGrand, Y. Liu, Phys. Rev. D94, 034506 (2016), [Erratum: Phys. Rev.D95,no.1,019902(2017)], 1606.01277 